\title{
ANSEYS DE MES
}

(corrections).

J'ai annoncé l'édition de l'Anseijs, publiée par M. H. J. Green, dans cette revue (XXVII, p. 146). Depuis j'ai eu l'occasion de relire ce texte, et j'ai le regret de dire que la mauvaise impression que j'ai reçue du travail de $M$. Green a été renforcée par cette nouvelle lecture. Malgré quelques timides corrections en partie fautives, et en dépit de quelques essais d'explication, en partie malvenus, on peut dire que cette édition nous présente à peu près le texte tel qu'il est sorti de la plume d'un scribe nonchalant, ou plutôt dans un état pire encore, étant publié par un éditeur insuffisamment préparé à cette tâche. Aussi ne me semble-t-il pas inutile de proposer une série de corrections que j'ai faites pendant ma lecture. Elles sont nombreuses, et pourtant il reste encore plusieurs passages obscurs à expliquer ou à émender.

1) Commençons par quelques cas où la difficulté se réduit à une question d'orthographe. On sait que $a$ nasal s'écrit indifféremment an ou en, de sorte qu'il faut interpréter enmastes, 736, comme amastes, et parenmai, 1183, comme paramai. Or, au vers 98 ,Or i mouvrai en nuit ou le matin", et au v. 14255 ,S'au logement voloit fere de mi Nos esterions ancore en nuit ami", il faut évidemment lire ennuit, c'est-à-dire antuit (hac nocte). Il faut de même au v. 152 ,En deus ses bras il a a son col mis" lier les deux premiers mots Endeus ou andeas, comme on le trouve correctement écrit au v. 11899. Lisons aussi en un seul mot enchaucent, 2519, et envoie, 5210. Par contre il vaut mieux scinder en deux parties s'entorne, 5612, 11247, 11248, 11364, puisqu'on trouve aussi s'en est tornez.

On sait que $s$ devant consonne s'est amule. De là les graphies itrons, 1678 (istrons), s'en voit (s'en voist), 7615 , s'en aille"; et, d'autre part lestre (letre), 2601, mestre (metre), 3423 , luiestes (luietes), 5960 ,petite lieue"; les costiaus du v. 4490 sont des couteaux, et reperi, 1652, est resperi du verbe esperir ,se réveiller, reprendre ses esprits". Il est donc clair que dans ,Mort le trebuche qui fut let ne qui bel" fut est pour fust, de même au vers $9180 \mathrm{fll}=$ fust, tandis que par contre au v. $11943 \mathrm{fust}=$ fut. , Or pent chascuns de son cors revengier", 2457 ; pent est naturellement penst ,que chacun pense”. Comme $s$ et $c$ ont la même prononciation, on n'est pas étonné de lire $S e$ cenefie, 427 , pour $\mathrm{Ce}$ senefie. Si donc aux vers $1631-1635$ on lit:

Si con Gerins l'aproche, li marchis

Choisit Hernaus son frere ceglentir.

De pamoison se redreça et vint,

Euvre les ieus, si regarde entort li.

nous interprétons ceglentir comme se glentir ou glantir ,clignoter”, et nous notons l'emploi dans notre texte de ce verbe, dont Godefroy donne seulement un exemple du quinzième siècle.

On sait aussi à quel point $s$ et $f$ se ressemblent dans les manuscrits et combien de fois des scribes négligents les ont confondus. Il n'est donc pas douteux qu'il faille changer de chies en chief, 5102, en de chiet en chief, tournure qui se trouve au v. 3971; c'est d'ailleurs une cheville qui corrompt la mesure du vers: ,Trestout l'afere li ont de chies en chief jehı e di'". Ainsi, des maufef vif est une faute évidente pour maufes vis, 8989 , et vif fier, 13342 , pour vis fier, et li fes Aloris pour li fel. On est donc en droit de substituer à la forme ie vis, aux vers $62,113,156,240$ et 470 , la forme primitive je vif, d'autant plus que celle-ci se lit réellement v. 1490.

La ressemblance entre ces deux lettres à fait commettre à $M$. Green une faute assez plaisante. Au v. 1959 nous nous trouvons dans l'église: „Li enfenz flere", ce qui signifierait, d'après M. Gr., "Les enfants pletrent”, et flere serait un infinitif historique latin, transplanté tel quel - mirabile dictu - dans une chanson de geste française! La vérité est qu'il faut lire: Li ensens (encens) flere (flaire). Le palis antis et $l i$ baron ententis des vers 290-291 enfin sont des formes dues au copiste désireux de remplacer l'assonance par la rime. Signalons encore la graphie esmes à côté de elmes, graphie qui s'explique, non tant par la ressemblance entre les deux caractères $l$ et $s$, que plutôt parce que dans elme, qui aura été prononcé comme iaume, $l$ n'ayant aucune valeur peut être remplacé facilement par $s$, qui n'en a pas davantage. 
Dans Achillet, 4511, Nievlet, 11601, et dans la locution a fait de fascem, 12279 et 12525, il s'agit d'une simple inadvertance de la part du scribe.

Les deux caractères qui se confondent peut-être le plus souvent sont $n$ et $u$. Il ne parait pas que 1'éditeur y ait suffisamment fait attention: a vostre ens, 3311, est évidemment a vostre eus (ues) de opus, lenee, 3200 , levee, reune, 4809, retve (rogat), asonage, 6582, asouage ,apaiser", truie, 8271, est trive (,trève"), forme qu'on lit 12810; la tor antine, 5561, est antive, et dans le vers 7236 „Buisines sonent et meunel ausi", où il manque une syllabe, il faut remplacer meunel par menuel ,petit cor".

Au v. 6579 et au v. 3452 , Renulle et Ril, l'éditeur aurait dû séparer les deux éléments et indiquer que la majuscule $\mathrm{R}$ est pour la majuscule $\mathrm{N}$, confusion assez fréquente dans les mss., et qu'il faut donc entendre Ne nulle et $N^{\prime} i l$. De même Renris, 8498, est pour Henris.

Une confusion fréquente est encore celle entre $c$ et $t$. Ainsi notre texte donne ponc pour pont, $11628,11780,11827$, porc pour port, 3238 et 3252 , fronc pour front, 4168 et 11136 , boc pour bos, 12343 et 12349 . Lisons donc Estes vos ciaus, 9395 , au lieu de Es ces vos ciaus, et Ernou le tirant, 9677 pour cirant, que l'éditeur traduit par ,candlemaker"?

Quelquefois le scribe a oublié de mettre un tiret au-dessus d'une voyelle pour indiquer qu'elle est suivie d'un $n$ : on aurait donc dû imprimer tre[n]chent et gue $[n]$ chis aux vers. 7396 et 7310. Au v. 8388, le combat recommence, ,Mais il enmassent assez mieus aloier Por la dolor qu'il ont de lor moillier. M. Green traduit aloier par ,to have intimate connection with a woman"; nous entendons alo[n]ier, c'est à dire aloignier ,s'éloigner". Il faut, par contre, biffer le $n$ dans l'expression bien connue $a$ coi[ $n] t e$ d'esperon ,en piquant des éperons", 6564 et 6495 .

2) Notre texte présente un assez grand nombre de vers boiteux, soit que le copiste ait sauté une syllabe ou un ou même plusieurs mots, soit qu'il ait remplacé la forme primitive par une autre plus récente. Au v. 6927 ,La veïssiez tan teste trenchier", l'éditeur a corrigé tan en tan[t]; il est évident qu'il faut lire tan[te] pour que le vers soit correct. Et pourquoi le savant américain n'a-t-il pas supprimé la voyelle finale de mile, oüe et grande aux vers $12000,12794,13118$, et pourquoi ne l'a-t-il pas ajoutée aux mots trench[e], 7301, s'espe[e], 7779 et 8532, mi[e], 13806 cr[e]antames, 10228, et la nois $[e], 6735$, où il ne s'agit pas de neige, mais du vacarme de la bataille. Et puisque l'auteur connaît les deux formes marbrin et marberin, com et come, encor et encore, et les deux futurs pendrai et penderai, plaindrai et plainderai, on aurait dû, selon les exigences du vers, mettre $e$ entre crochets ou entre parenthèse dans les nombreux passages où on lit une de ces formes; de même duera, 2607, alterne avec dura, 2614, entendez durra = durera.

Le scribe a souvent ajouté un $s$ là où l'original ne l'avait pas. On rétablit le rythme du vers en biffant cet $s$ dans sire(s), 7115, esme(s), 6097, Bauche(s), 5571 et 9014, jus$q u e(s), 7945$. Pour la même raison nous devons lire ga[a]gnier, 3128 et 10314 , ba [a]illier, 12240 , tandis que nous pouvons hésiter entre chaignnon et cha[a]ignon au v. 12168. C'est encore en rétablissant la forme primitive qu'on obtient un vers correct dans: pre[e]scha, 5083, di $[e], 3320$, ne $l[e], 1917$, estov[e]roit, 5280, Sal[o]mons, 9031, 6561, or [i] flambe, 6733, Niev[e]les, 11640, aïr[er], 11686, commence[nt], 13739, n[en] ait, 4009, [en] jura, 2656, [ce] puet estre, 2566, [la] mer salee, 5527, [li] sires de Lisignon, 5918.

Au v. 5603 , Por lor amis, ce dient, vengier, qui sont en terre”, il faut biffer ce dient, mots qui se trouvent aussi au vers précédent. Dans „,De la bataille li conta ensement ou vaincu fumes si malement", 4776, la première personne du pluriel fumes étonne, il manque en outre une syllabe. Je propose donc de lire ,Ou vaincu fu, [ja] mes si malement". Il est enfin facile de corriger le v. 10102 „De vostre pere ne vos sovient [il] mie".

3) Ici, nous voudrions ranger un certain nombre de passages qui demandent à être corrigés. Tout au commencement de la chanson Hernaut a appris la mort de Fromondin, puis il est rentré à Gironville.

A Gironville fu Hernaus li gentis.

Et la contesse sa fame Ludiis,

Tot l'errement que il avoit apris... v. 46-48. 
Ces vers n'offrent pas de sens satisfaisant, et l'éditeur n'ajoute aucun commentaire. Nous proposons de lire: „Et la (= là) conta sa fame Ludiis"

Et ces paroles que tu avant tresis

Comparastras se Dieus plest et je vis, v. 239-240.

L. compar[r]as ja; cf. 1733 ,tu le comparas chier”.

En covent tout li petis Anceïs, v. 1246.

Il faut corriger En covent ( $t$ )out, ,,'est engage", tournure qui est reprise quatre vers plus loin ,Vos ot covent".

Les navrez font aferir et garir, v. 1605.

L. ajetir = afetier , panser".

La fet murir son parin Anseys,

Enbaucemer de chier baume de pris, v. 2036-7.

murir n'a pas de sens. On ouvre le corps pour l'embaumer; ainsi on lit v. 573 ,A un coutel a fet son pere ouvrir". Changeons donc le $m$ de murir en $o, u$ ou ou, et lisons ouvrir.

Vos dites voir que nos Bordelle prise, v. 4239.

On obtiendra le verbe manquant en lisant quavōs, c'est à dire qu'avons.

L'ui tiers sanz plus pencé d'esperonner, v. 5347.

Il s'agit de la tournure Lui tiers, cf. Foulet, Romania, 1936, p. 29-39.

Ou lienas a fet tendre ses trez, v. 5796.

C'est Ludias qui dresse ses tentes.

Mort le trebuche, si en gaingne li mons, v. 7714.

L'auteur ne s'est pas servi du verbe gaignier, ou plutôt gaaignier, mais de engraignier, „grandir". Nous lisons „,Si eng[r]aingne li mons".

Armes qu'il ait ne li vaut un voisin. v. 8138 .

Voisin n'a que faire ici; le poète a dit roisin ,raisin".

Adonc s'aïrent François et Brebençon.

Ensaignent, crient entor et environ. v. 8343-4.

Ils n'enseignent rien, mais ils poussent leur cri de ralliement: Ensaigne crient, cf. commence(nt), 13739.

Dans la bataille sévit une terrible virago, qui armée d'une lourde massue écrase les rangs ,et devant et derriere"; c'est ,une grande hemmere", 8719. Ce dernier mot est un dérivé de homme, le changement de la voyelle ne nous étonne pas (cf. domage et damage); il faut seulement lire hemmiere, forme réclamće par la rime. Le mot, qui a la même valeur que homesse, ne se trouve pas dans Godefroy.

Se sui connus par aucune conaissance

Ja en Gascoigne ne ferons retornance. v. 9290-1.

déclare Anseys, qui se trouve en pays ennemi. Le premier vers a une syllabe de trop, mais cette fois la faute n'est est pas au scribe, mais à l'éditeur, car le ms. a en aisance, "which I interpreted as meaning: on the way out, in an exit or passage-way". Comme cette explication ne lui plaisait pas beaucoup, M. Gr. a mis dans le texte conaissance, ne voyant pas que par là il corrompait le vers. Il me semble qu' il faut lire "Se sui connus pas aucun(e) en aissance", et traduire "Si quelqu' un me reconnaït de près". En aisance aura donc le même sens que en aise, qu'on lit Eneas, 7149 (a aise, 7690), et nous notons cette valeur inconnue jusqu'ici.

Signalons encore quelques fautes de moindre importance: En pieu[r] lieu, 8101; Pau pour Pax, 9025 (le lecteur aura prononcé paus!), 
Estiens est à condamner, parce que c'est un pluriel, parce que c'est un passé, parce que c'est un mot trissylabique. Lisons estois.

sanz damor, 12420, est naturellement sanz demor ,,sans tarder", comme menier, 13678, est manier ,habile".

Il (Dieu) vos desfende que ne vos praigne mie.

Tost vos aroient l'ame du cors partie. v. 11332-3.

Comme le verbe de la première phrase manque de sujet, je crois qu'il faut lire quē ne, c'est à dire "qu'en ne vos praigne mie", qu'on ne vouts prenne pas".

Nous terminerons cette longue série de critiques par deux passages assez intéressants:

Cis rois de France n'a mie consoil bel

Qui ainsi cuide abatre nos flavel. v. $4979-80$.

L'éditeur, qui dans Lacurne de Sainte Palaye a trouvé flavel au sens de ,éventail", "flatterie" au figuré, croit que ,abatre nos flavel" signifie ,to reject our homage, or conciliatory efforts". Il me semble pourtant que flavel est une autre forme pour flavele, favele, et que abatre no(s) flavel équivaut donc exactement à la locution moderne "rabattre le caquet".

Bauche, qui voudrait empêcher la guerre sanglante,

...en deprie chascun jor envers Dieu

Qui pais i meste ainz qu'i soient fet Dieu. v. 5004-5.

, avant qu'ils soient faits Dieu". On se demande ce que cela pourrait bien signifier; l'éditeur ne donne aucun commentaire. Il est pourtant clair qu'il faut lire fetdieu, c'est à dire faidieu ou faidif ,ennemis mortels". Je rappelle encore la bévue de l'éditeur, qui croit que au maille, 11282 , est une locution négative, tandis que nous avons simplement affaire au substantif aumaille ,bétail"; cf. Neoph., XXVII, p. 147.

Pour publier un texte du vieux français, il n'est pas inutile de posséder une certaine connaissance de la langue et de la paléographie et d'être doué d'un certain acumen philologicum.

Groningen.

K. SNEYders de VOGEL.

\section{DIE BITTE DER KÖNIGIN UND DAS HIRSCHKOPF- ODER KUSZMOTIV IM EREC.}

Artur will den weißen Hirsch jagen. Es ist die Sitte, daß derjenige, der den Hirsch niederstreckt, die schönste Dame des Hofes küssen darf: Chrestien 36 ff. ${ }^{1}$ ). Das Mabinogi Geraint ab Erbin ${ }^{2}$ ) hat statt des KuBmotivs das Hirschkopf-motiv: der glückliche Jäger darf der schönsten Dame den Kopf des Hirsches anbieten. Nachdem Artur den Hirsch getötet hat, ersucht ihn aber die Königin, mit dem Kuße, bzw. mit dem Überreichen des Hirschkopfes zu warten, bis Erec, der sich auf einer Rachefahrt befindet, zurück sei. Der König gewährt ihr diese Bitte. Erec kommt mit der schönen Enite zurück, die dann zur schönsten Dame erklärt wird.

Es erheben sich zwei Fragen: ist das Hirschkopfmotiv (Mab.) oder das Kußmotiv (Chres. und Hartmann von Aue) ursprünglich? und: ist die Bitte der Königin nicht in allen Fassungen widersinnig? Denn, wie soll man vorher wissen, daß Erec die schöne Enite mitbringen wird!

Sparnaay ${ }^{3}$ ) glaubt die Widersinnigheit durch die Annahme zu beheben, daß die Reihenfolge der Motive im Mabinogi einmal so gewesen sei, daß der Ritter, an dem sich Erec gerächt habe und von dem man erfahre, daß Erec mit Enite, für die dieser im Sperberkampf gesiegt habe, auf dem Wege nach dem Hofe sei, daß also dieser Ritter, Edern genannt, an den Hof komme, bevor Guenievre ihre Bitte vorbringe. Bei Chrestien kommt Edern erst viel später an den Hof. Damit wir d Chrestiens Fassung noch widersinniger. Das Hirschkopfmotiv wird als das ältere betrachtet, namentlich, weil es $n$ der Percevalfortsetzung von Gautier, im Didot-Perceval und im Bel Desconu vorkommt und nach Gaston Paris ${ }^{4}$ ) die Sitte in Frankreich noch heute besteht, das

1) W. Foerster, Kleine Erecausgabe, Halle 1934.

2) J. Loth, Les Mabinogion, Paris 1889. Die 2. Auflage 1913 ist mir unerreichbar.

3) $Z$. f. rom. Phil., XLV, 53 ff. und Hartmann von Aue, Halle 1933, I, S. 69 ff.

) Romania, XX, 156. 